\title{
Correction to: Thermal, spectroscopic and antimicrobial activity characterization of some norfloxacin complexes
}

\author{
Wilhan Donizete Gonçalves Nunes ${ }^{1} \cdot$ André Luiz Carneiro Soares do Nascimento $^{1}$ - Aniele Moura ${ }^{1}$. \\ Caroline Gaglieri ${ }^{2} \cdot$ Gustavo Brunelli Vallim $^{3} \cdot$ Luiz Carlos Nascimento $^{3} \cdot$ Roni Antônio Mendes $^{4}$. \\ Massao lonashiro ${ }^{1}$. Flávio Junior Caires ${ }^{1,2}$
}

Published online: 21 February 2018

(C) Akadémiai Kiadó, Budapest, Hungary 2018

\section{Correction to: Journal of Thermal Analysis and Calorimetry https://doi.org/10.1007/s10973-018-7019-z}

In the original publication, the fifth author's given name was misspelled as "Gabriel". The corrected name is given in this erratum. The original article has been corrected.

The original article can be found online at https:// doi.org/10.1007/s10973-018-7019-z.

Flávio Junior Caires

caires.flavio@yahoo.com.br; caires.flavio@fc.unesp.br

Wilhan Donizete Gonçalves Nunes

wdgns1@gmail.com

André Luiz Carneiro Soares do Nascimento

nascimento.a.1.c@gmail.com

Aniele Moura

m.aniele@yahoo.com.br

Caroline Gaglieri

carolinegaglieri@fc.unesp.br

Gustavo Brunelli Vallim

gustavobvallim@gmail.com

Luiz Carlos Nascimento

luiz.nascimento@unifal-mg.edu.br

Roni Antônio Mendes

roni.mendes@unifal-mg.edu.br
Massao Ionashiro

massaoi@yahoo.com.br

1 Instituto de Química, Universidade Estadual Paulista (UNESP), Araraquara, SP, Brazil

2 Faculdade de Ciências, Universidade Estadual Paulista (UNESP), Bauru, SP, Brazil

3 Faculdade de Ciências Farmacêuticas, Universidade Federal de Alfenas (UNIFAL-MG), Alfenas, MG CEP 37130-001, Brazil

4 Instituto de Ciência e Tecnologia, Universidade Federal de Alfenas (UNIFAL-MG), Poços de Caldas, MG CEP 37715-400, Brazil 